\title{
Planar-Based Visual Inertial Navigation
}

\author{
Ghazaleh Panahandeh* and Peter Händel ${ }^{\triangleright}$ \\ ${ }^{*}$ KTH Royal Institute of Technology, ACCESS Linnaeus Center, Stockholm, Sweden \\ Volvo Car Corporation, Gothenburg, Sweden \\ ${ }^{\diamond}$ KTH Royal Institute of Technology, ACCESS Linnaeus Center, Stockholm, Sweden
}

\begin{abstract}
This paper investigates the problem of visual-inertial navigation. The proposed navigation system integrates inertial information from an inertial measurement unit (IMU) with visual data from a camera to provide relative pose estimation for a system which is navigating in an unknown structural environment. The main contribution of this paper is derivation of a novel measurement model based on inertial data and visual planar features. The proposed formulation is a solution to the 6-DoF motion estimation where the IMU-camera movement is not restricted over a desired navigation plane. Compared to previous works, which are restricted on using only horizontal plane features, the proposed model is generalized for arbitrary planar features. The theoretical finding of this study is extensively evaluated both with simulation and real world experiments. The presented experiments indicate the reliability of the proposed method to perform accurate 6-DoF pose estimation.
\end{abstract}

\section{INTRODUCTION}

Recently, there has been growing research interest towards visual-inertial navigation systems, where the hardware structure consists of an inertial measurement unit (IMU) and a monocular camera. Popularity of these systems is especially due to the rapid improvements of smart devices with standard motion capture sensors, using which a low-cost navigation solution can be designed. The visual-inertial navigation systems have been already implemented in commercial devices such as industrial robots, smart phones, smart cars, or smart delivery devices such as Amazon prime air. The current research efforts are mainly focused on having better sensor fusion strategies for more accurate positioning systems with less computational complexity.

In principle, a 6-DoF motion estimation method can be designed by integrating the linear accelerations and rotational velocities measured using an IMU. However in such method that is only based on the inertial information, the system noise results in integration drift, and hence, the estimated positions and rotations must be periodically corrected; otherwise, the system cannot be used over an extended period of time. In visual-aided inertial navigation systems, the correction is provided by camera images. The correction from the images can be provided in different forms such as feature point positions, optical flow, vanishing points or lines.

In this paper, we introduce a novel visual-inertial integration approach where visual features on a plane are used for motion estimation. The plane-facing cameras can provide a reliable visual information when navigating in environments with poor features or regions with dynamic backgrounds. One interesting 978-1-4799-9858-6/15/\$31.00 (C) 2015 IEEE application of such system is for precise planetary landing in the spacecraft control systems [1]. Other applications include, e.g., indoor cleaning robots [2], automated parking systems [3], and industrial robots [4] where most of the actions are performed in front of a planar surface.

The proposed navigation system is formulated in a state space model and the pose estimation is performed using a nonlinear Kalman filter. The key contribution of this work is the derivation of a novel nonlinear measurement equation in which 3D positions of the planar features and their projections in the image plane are related to the $3 \mathrm{D}$ pose of the IMUcamera. The theoretical derivations are then evaluated through extensive simulations and experiments and it is shown that the proposed system is capable of achieving relatively good results when using only a few visual features.

Previously, the observation of planar features of the ground plane was implicitly used in different visual inertial navigation systems, such as [1], [5]-[8]. Several methods have been proposed in [9], where the properties of the planar features on a horizontal plane are explicitly used to derive the system state-space model; advantages and different aspects in this visual-inertial navigation system including motion estimation, horizontal plane feature detection, and observability analysis have been also addressed in [9]. In contrast to the earlier works, the proposed method in this paper is not restricted to horizontal planar features, but rather it is generalized for arbitrary planar features. This opens up for new applications where the plane under navigation is not horizontal but rather tilted or vertically aligned, e.g., in robotics [10].

\section{A. Notation}

In the following, some of the important notations are introduced.

- Scalars are denoted by lowercase letters $(s)$, vectors by bold letters (f), and matrices by bold capitals $(\mathbf{K})$.

- $\mathbf{I}_{m}$ denotes the $m \times m$ square identity matrix. $\mathbf{0}_{m \times n}$ denotes the $m \times n$ zero matrix. $\mathbf{e}_{i} \in \mathbb{R}^{3}$ for $i=1,2,3$ and $\mathbf{e}_{1}=$ $\left[\begin{array}{lll}1 & 0 & 0\end{array}\right]^{\top}, \mathbf{e}_{2}=\left[\begin{array}{lll}0 & 1 & 0\end{array}\right]^{\top}$, and $\mathbf{e}_{3}=\left[\begin{array}{lll}0 & 0 & 1\end{array}\right]^{\top}$, where $\top$ denotes the transpose operator.

\section{PRopagation MODEL}

The goal of the proposed method is to estimate the pose of a mobile visual inertial navigation system with respect to a reference frame, denoted by $\{G\}$. The hardware structure consists of a camera rigidly mounted on an IMU. To preserve the clarity of the presentation, we assume that the IMU and the camera 
frame of reference coincide, that is $\left\{{ }^{I} \mathbf{p}_{C}=\mathbf{0}_{3 \times 1}, \mathbf{C}\left({ }^{I} \mathbf{q}_{C}\right)=\mathbf{I}_{3}\right\}$. Thereby, variables are expressed in camera frame of reference when refereing to the camera or the IMU coordinate frames.

For this system, we define the inertial navigation system (INS) state variables in the form of the state vector as

$$
\mathbf{x}^{\text {ins }}=\left[\begin{array}{lllll}
{ }^{C} \boldsymbol{\theta}_{G}{ }^{\top} & { }^{G} \mathbf{v}_{C}{ }^{\top} & { }^{G} \mathbf{p}_{C}{ }^{\top} & \mathbf{b}_{a}^{\top} & \mathbf{b}_{g}^{\top}
\end{array}\right]^{\top},
$$

where ${ }^{C} \theta_{G} \in \mathbb{R}^{3 \times 1}$ represents the orientation of the global frame $\{G\}$ in the camera's frame of reference $\{C\}$, [11]. The velocity and the position of the camera in the global frame are denoted by ${ }^{G} \mathbf{v}_{C}$ and ${ }^{G} \mathbf{p}_{C}$, respectively. $\mathbf{b}_{a}$ and $\mathbf{b}_{g}$ are the bias vectors affecting the accelerometer and gyroscope measurements, respectively. The position, velocity, and the attitude in this model are estimated at a high rate given noisy IMU measurements, i.e., accelerometers and gyroscopes signals.

The time evolution of the INS state is given by

$$
\begin{aligned}
{ }^{C} \dot{\mathbf{q}}_{G}(t) & =\frac{1}{2}\left[\begin{array}{cc}
-\lfloor\omega(t)\rfloor & \omega(t) \\
-\omega(t) & 0
\end{array}\right]{ }^{C} \mathbf{q}_{G}(t), \\
{ }^{G} \dot{\mathbf{v}}_{C}(t) & ={ }^{G} \mathbf{a}(t)={ }^{G} \mathbf{g}+\mathbf{C}\left({ }^{C} \mathbf{q}_{G}(t)\right){ }^{\top}\left(\mathbf{a}(t)-\mathbf{b}_{a}(t)\right), \\
{ }^{G} \dot{\mathbf{p}}_{C}(t) & ={ }^{G} \mathbf{v}_{C}(t), \quad \dot{\mathbf{b}}_{a}(t)=\mathbf{n}_{\delta a}, \quad \dot{\mathbf{b}}_{g}(t)=\mathbf{n}_{\delta g},
\end{aligned}
$$

where $\omega(t)=\left[\begin{array}{lll}\omega_{1} & \omega_{2} & \omega_{3}\end{array}\right]^{\top}$ and $\mathbf{a}(t)=\left[\begin{array}{lll}a_{1} & a_{2} & a_{3}\end{array}\right]^{\top}$ are the IMU rotational velocity and linear acceleration, ${ }^{G} \mathbf{g}=$ $\left[\begin{array}{lll}0 & 0 & g\end{array}\right]^{\top}$ is the gravitational acceleration, $\mathbf{C}(\mathbf{q})$ is the rotation matrix corresponding to quaternion $\mathbf{q} \in \mathbb{R}^{4 \times 1}$, and $\mathbf{n}_{\delta a}$ and $\mathbf{n}_{\delta g}$ are the accelerometer and gyroscope biases driving white Gaussian noises. The output measurement signals of the gyroscope, $\omega_{m}$, and accelerometer, $a_{m}$, are modeled as

$$
\begin{aligned}
\omega_{m}(t) & =\omega(t)+\mathbf{b}_{g}(t)+\mathbf{n}_{g}(t), \\
a_{m}(t) & =\mathbf{C}\left({ }^{C} \mathbf{q}_{G}(t)\right)\left({ }^{G} \mathbf{a}(t)-{ }^{G} \mathbf{g}\right)+\mathbf{b}_{a}(t)+\mathbf{n}_{a}(t) .
\end{aligned}
$$

To construct the state space model of the system, we start with the process equations which are derived based on INS propagation models (2) for the discrete-time model. This model is derived based on the standard additive error definition for the position, velocity, and biases $(\delta x \simeq x-\hat{x})$, and quaternion error for the rotational Euler angles $\left(\delta \mathbf{q} \simeq\left[1 \frac{\theta}{2}\right]^{\top}\right)$, see [9] for derivations. We define the INS error state vector as

$$
\delta \mathbf{x}^{\text {ins }}=\left[\begin{array}{lllll}
\delta^{C} \boldsymbol{\theta}_{G}{ }^{\top} & \delta^{G} \mathbf{v}_{C}^{\top} & \delta^{G} \mathbf{p}_{C}^{\top} & \delta \mathbf{b}_{a}^{\top} & \delta \mathbf{b}_{g}^{\top}
\end{array}\right]^{\top} .
$$

Then the nonlinear INS propagation model in the form of a discrete-time linear state-space model for a period of $\delta t$ is

$$
\delta \mathbf{x}_{k+1}^{\text {ins }}=\mathbf{F}_{k}^{\text {ins }} \delta \mathbf{x}_{k}^{\text {ins }}+\mathbf{G}_{k}^{\text {ins }} \mathbf{n}_{k} \in \mathbb{R}^{15},
$$

where $\mathbf{F}_{k}^{\mathrm{ins}}$ and $\mathbf{G}_{k}^{\mathrm{ins}}$ are known as discrete time state and system noise propagation matrices, respectively:

$\mathbf{F}_{k}^{\text {ins }}=\left[\begin{array}{ccccc}\mathbf{I}_{3} & \mathbf{0}_{3 \times 3} & \mathbf{0}_{3 \times 3} & \mathbf{0}_{3 \times 3} & -d t \mathbf{C}\left({ }^{C} \hat{\mathbf{q}}_{G}\right) \\ \left.d t \mid \mathbf{C}\left({ }^{C} \hat{\mathbf{q}}_{G}\right) \hat{\mathbf{a}}\right\rfloor & \mathbf{I}_{3} & \mathbf{0}_{3 \times 3} & d t \mathbf{C}\left({ }^{C} \hat{\mathbf{q}}_{G}\right) & \mathbf{0}_{3 \times 3} \\ \mathbf{0}_{3 \times 3} & d t \mathbf{I}_{3} & \mathbf{I}_{3} & \mathbf{0}_{3 \times 3} & \mathbf{0}_{3 \times 3} \\ \mathbf{0}_{3 \times 3} & \mathbf{0}_{3 \times 3} & \mathbf{0}_{3 \times 3} & \mathbf{I}_{3 \times 3} & \mathbf{0}_{3 \times 3} \\ \mathbf{0}_{3 \times 3} & \mathbf{0}_{3 \times 3} & \mathbf{0}_{3} & \mathbf{0}_{3 \times 3} & \mathbf{I}_{3}\end{array}\right]$

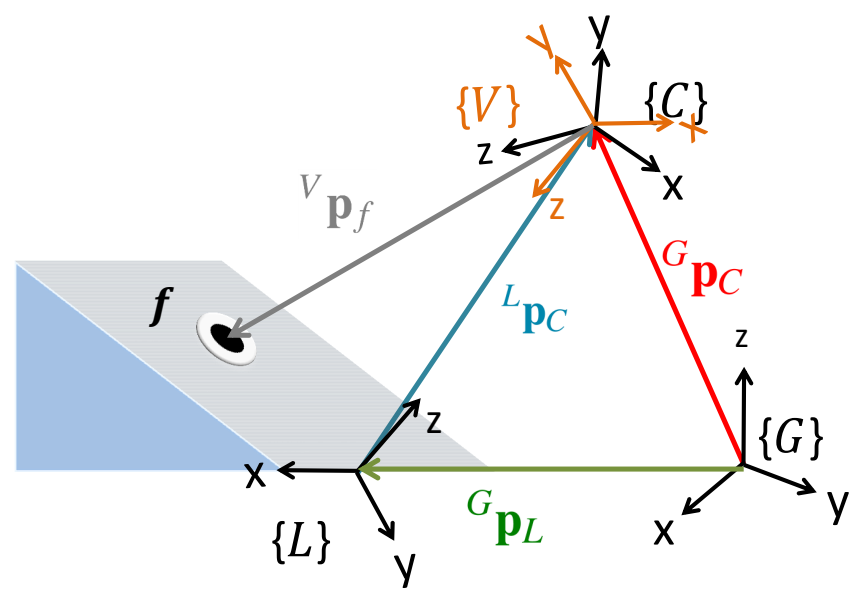

Fig. 1: Graphical representation of the system. The camera and the virtual camera coordinate frames are depicted by $\{C\}$ and $\{V\}$, respectively. The optical axis of the virtual camera is selected to be always orthogonal to the desired plane. The global coordinate frame $\{G\}$ is located on the horizontal plane. The local coordinate frame $\{L\}$ is located on the intersection of the global frame and the desired navigation frame, such that $\mathbf{e}_{3}^{\top G} \mathbf{p}_{L}=0$, where its $z$ axis is orthogonal to the desired plane.

and

$$
\mathbf{G}_{k}^{\text {ins }}=\left[\begin{array}{cccc}
\mathbf{0}_{3 \times 3} & -d t \mathbf{C}\left({ }^{C} \hat{\mathbf{q}}_{G}\right) & \mathbf{0}_{3 \times 3} & \mathbf{0}_{3 \times 3} \\
d t \hat{\mathbf{C}} & \mathbf{0}_{3 \times 3} & \mathbf{0}_{3 \times 3} & \mathbf{0}_{3 \times 3} \\
\mathbf{0}_{3 \times 3} & \mathbf{0}_{3 \times 3} & \mathbf{0}_{3 \times 3} & \mathbf{0}_{3 \times 3} \\
\mathbf{0}_{3 \times 3} & \mathbf{0}_{3 \times 3} & d t \mathbf{I}_{3} & \mathbf{0}_{3 \times 3} \\
\mathbf{0}_{3 \times 3} & \mathbf{0}_{3 \times 3} & \mathbf{0}_{3 \times 3} & d t \mathbf{I}_{3}
\end{array}\right] .
$$

$\mathbf{n}_{k}=\left[\begin{array}{llll}\mathbf{n}_{a}^{\top} & \mathbf{n}_{g}^{\top} & \mathbf{n}_{\delta a}^{\top} & \mathbf{n}_{\delta g}^{\top}\end{array}\right]^{\top}$ is the process noise (assumed to be zero-mean wide-sense stationary) with the corresponding diagonal covariance matrix $\mathbf{Q} \in \mathbb{R}^{15 \times 15}, \mathbf{C}\left({ }^{C} \hat{\mathbf{q}}_{G}\right)$ is the estimated rotation matrix, and $\hat{\mathbf{a}}=\mathbf{a}_{m}-\hat{\mathbf{b}}_{a}$, see [9] for details.

\section{Camera Measurement Model}

In Section III-A, we first derive the projection model of the camera in terms of state variables and the $3 \mathrm{D}$ position of the features on the desired navigation plane, Section III-A. Then in Section III-B, the relative transformation of the camera between two views is derived using the proposed projection model. Based on the derivations in the preceding sections, the camera measurement model is introduced in Section III-C.

\section{A. Projection model}

Consider a feature point $f$ observed by a camera, where its position relative to the camera center $\{C\}$ is denoted by ${ }^{C} \mathbf{p}_{f}$. Using the pinhole camera model [12], its homogenous and normalized pixel coordinates on the image plane are represented by ${ }^{C} \overline{\mathbf{z}}$ and ${ }^{C} \mathbf{z}$, respectively, as

$$
\begin{gathered}
{ }^{C} \overline{\mathbf{z}}=\left(\mathbf{e}_{3}^{\top C} \mathbf{p}_{f}\right)^{-1 C} \mathbf{p}_{f}, \\
{ }^{C} \mathbf{z}=\left[\begin{array}{ll}
\mathbf{I}_{2} & \mathbf{0}_{2 \times 1}
\end{array}\right]\left(\mathbf{e}_{3}^{\top C} \mathbf{p}_{f}\right)^{-1 C} \mathbf{p}_{f}, \\
{ }^{C} \mathbf{p}_{f}=\mathbf{C}\left({ }^{C} \mathbf{q}_{G}\right)\left({ }^{G} \mathbf{p}_{f}-{ }^{G} \mathbf{p}_{C}\right) .
\end{gathered}
$$


Lemma 1: For an arbitrary feature point $f$ on a desired plane, the camera projective model can be represented as

$$
{ }^{C} \overline{\mathbf{z}}=\frac{-\mathbf{e}_{3}^{\top} \mathbf{C}\left({ }^{L} \mathbf{q}_{C}\right)^{C} \overline{\mathbf{z}}_{C}}{\mathbf{e}_{3}^{\top} \mathbf{C}\left({ }^{L} \mathbf{q}_{G}\right){ }^{G} \mathbf{p}_{C}} \mathbf{p}_{f} .
$$

Proof: Let us consider a virtual camera along with our real camera in which the center of its coordinate frame $\{V\}$ coincides with the center of the real camera coordinate frame $\{C\}$. Moreover, the virtual camera optical axis is always restricted to be orthogonal to the desired plane, see Fig. 1.

As the transformation between the real camera frame and the virtual frame is related by a rotation, ${ }^{C} \mathbf{C}_{V} \triangleq$ $\mathbf{C}\left({ }^{C} \mathbf{q}_{G}\right) \mathbf{C}\left({ }^{V} \mathbf{q}_{G}\right)^{\top}$, the positions of feature point $f, \mathbf{p}_{f}$, in the two coordinate frames are related by

$$
{ }^{C} \mathbf{p}_{f}={ }^{C} \mathbf{C}_{V}{ }^{V} \mathbf{p}_{f} .
$$

Substituting ${ }^{V} \mathbf{p}_{f}$ in (13) with its projection in the virtual image plane (9), we get

$$
{ }^{C} \mathbf{p}_{f}={ }^{C} \mathbf{C}_{V} \mathbf{e}_{3}^{\top V} \mathbf{p}_{f}{ }^{V} \overline{\mathbf{z}}
$$

Moreover, similar to (13), the projection of feature point $f$ in the virtual image plane can be mapped to the real image plane as

$$
C_{\overline{\mathbf{z}}}=\alpha^{C} \mathbf{C}_{V}^{V} \overline{\mathbf{z}}
$$

in which the equality holds only up to a scale factor $\alpha$. Then, by substituting ${ }^{V} \overline{\mathbf{z}}$ from (15) into (14), we get

$$
\begin{aligned}
{ }^{C} \mathbf{p}_{f} & =\alpha^{-1} \mathbf{e}_{3}^{\top V} \mathbf{p}_{f}{ }^{C} \mathbf{C}_{V}{ }^{C} \mathbf{C}_{V}{ }^{\top}{ }^{C} \overline{\mathbf{z}} \\
& =\alpha^{-1} \mathbf{e}_{3}^{\top V} \mathbf{p}_{f}{ }^{C} \overline{\mathbf{z}},
\end{aligned}
$$

or in other representation

$$
{ }^{C} \overline{\mathbf{z}}=\frac{\alpha}{\mathbf{e}_{3}^{\top V} \mathbf{p}_{f}}{ }^{C} \mathbf{p}_{f} .
$$

From (15) and given that the optical axis of the virtual camera is orthogonal to our desired plane, $\alpha$ can be written as

$$
\begin{aligned}
\alpha & =\mathbf{e}_{3}^{\top{ }^{C}} \mathbf{C}_{V}{ }^{\top}{ }^{C} \overline{\mathbf{z}}=\mathbf{e}_{3}^{\top} \mathbf{C}\left({ }^{V} \mathbf{q}_{L}\right) \mathbf{C}\left({ }^{L} \mathbf{q}_{G}\right) \mathbf{C}\left({ }^{C} \mathbf{q}_{G}\right){ }^{\top}{ }^{C} \overline{\mathbf{z}} \\
& =-\mathbf{e}_{3}^{\top} \mathbf{C}\left({ }^{L} \mathbf{q}_{G}\right) \mathbf{C}\left({ }^{C} \mathbf{q}_{G}\right){ }^{\top}{ }^{C} \overline{\mathbf{z}} \\
& =-\mathbf{e}_{3}^{\top} \mathbf{C}\left({ }^{L} \mathbf{q}_{C}\right){ }^{C} \overline{\mathbf{z}} .
\end{aligned}
$$

The next step is to derive $\mathbf{e}_{3}^{\top V} \mathbf{p}_{f}$ (from (16)) as a function of state or known variables. Let us consider a local coordinate frame $\{L\}$ on the intersection of desired plane and horizontal plane, such that its $\{z\}$ axis is the normal of the plane, see Fig. 1. Therefore the orientation of the virtual camera frame with respect to the local coordinate frame is $\mathbf{C}\left({ }^{V} \mathbf{q}_{L}\right)=\operatorname{diag}(1,-1,-1)$, and consequently

$$
\mathbf{e}_{3}^{\top V} \mathbf{p}_{f}=\mathbf{e}_{3}^{\top L} \mathbf{p}_{V}=\mathbf{e}_{3}^{\top L} \mathbf{p}_{C}
$$

From Fig. 1, it can be followed that ${ }^{L} \mathbf{p}_{C}$

$$
{ }^{L} \mathbf{p}_{C}=\mathbf{C}\left({ }^{L} \mathbf{q}_{G}\right){ }^{G} \mathbf{p}_{C}+{ }^{G} \mathbf{p}_{L},
$$

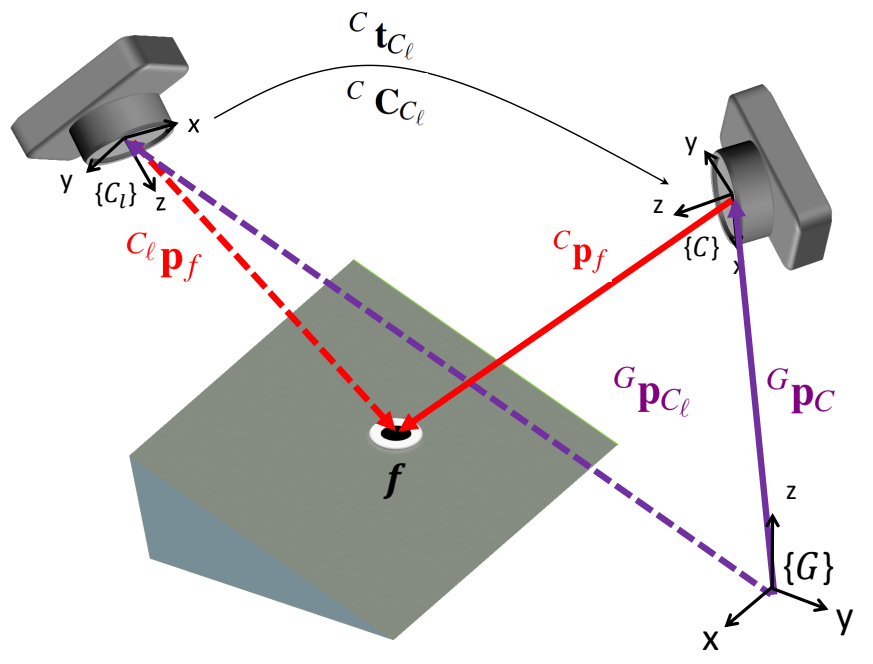

Fig. 2: The IMU-camera sensor unit system and its corresponding coordinate frames at current $\{C\}$ time and $\ell$ lags from the current one $\left\{C_{\ell}\right\}$. The relative rotation and translation between the time instants are denoted as ${ }^{C} \mathbf{C}_{C_{\ell}}$ and ${ }^{C} \mathbf{t}_{C_{\ell}}$, respectively. The global frame of reference $\mathrm{G}$ is assumed to be located on a horizontal plane, where its $z$ axis is orthogonal to the horizontal plane. A sample feature point $f$, located on the desired plane, is considered to be in the cameras' field of view.

then by multiplying $\mathbf{e}_{3}^{\top}$ to both side of (20), we have

$$
\begin{aligned}
\mathbf{e}_{3}^{\top L}{ }^{L} \mathbf{p}_{C} & =\mathbf{e}_{3}^{\top} \mathbf{C}\left({ }^{L} \mathbf{q}_{G}\right){ }^{G} \mathbf{p}_{C}+\mathbf{e}_{3}^{\top G}{ }^{G} \mathbf{p}_{L} \\
& =\mathbf{e}_{3}^{\top} \mathbf{C}\left({ }^{L} \mathbf{q}_{G}\right){ }^{G} \mathbf{p}_{C},
\end{aligned}
$$

and from (19), we can conclude that $\mathbf{e}_{3}^{\top V} \mathbf{p}_{f}=\mathbf{e}_{3}^{\top} \mathbf{C}\left({ }^{L} \mathbf{q}_{G}\right)^{G} \mathbf{p}_{C}$. By substituting this equality and $\alpha$ from (18) in to (17), we have

$$
C_{\overline{\mathbf{z}}}=\frac{-\mathbf{e}_{3}^{\top} \mathbf{C}\left({ }^{L} \mathbf{q}_{C}\right){ }^{C} \overline{\mathbf{z}}_{C}}{\mathbf{e}_{3}^{\top} \mathbf{C}\left({ }^{L} \mathbf{q}_{G}\right){ }^{G} \mathbf{p}_{C}} \mathbf{p}_{f} .
$$

It is worth mentioning that $\mathbf{e}_{3}^{\top} \mathbf{C}\left({ }^{L} \mathbf{q}_{G}\right)$ represents the normal of the plane with respect to $\{G\}$. The normal of the desired plane can be intuitively inferred from the gravity direction if the desired plane is aligned horizontally or vertically. To measure this value, one can use a clinometer, which is an instrument for measuring angles of slope with respect to gravity. More discussion is given in Section VI.

\section{B. Geometric constraint of the planar features}

Let us consider an arbitrary feature point $f$, which is in both $\left\{C_{\ell}\right\}$ and $\{C\}$ field of view. The geographical representation of this model is illustrated in Fig. 2. Following the representations and notations of Fig. 2, the position of $f$ with respect to $\left\{C_{\ell}\right\}$ and $\{C\}$ is given by

$$
{ }^{C} \mathbf{p}_{f}={ }^{C} \mathbf{C}_{C_{\ell}}{ }^{C} \mathbf{p}_{f}+{ }^{C} \mathbf{t}_{C_{\ell}},
$$

where

$$
\begin{gathered}
{ }^{C} \mathbf{C}_{C_{\ell}} \triangleq \mathbf{C}\left({ }^{C} \mathbf{q}_{G}\right) \mathbf{C}\left({ }^{C_{\ell}} \mathbf{q}_{G}\right)^{\top}, \\
{ }^{C} \mathbf{t}_{C_{\ell}} \triangleq \mathbf{C}\left({ }^{C} \mathbf{q}_{G}\right)\left({ }^{G} \mathbf{p}_{C_{\ell}}-{ }^{G} \mathbf{p}_{C}\right) .
\end{gathered}
$$

Equation (23) enables us to relate the observation of feature $f$ in two camera views to their corresponding translations, 
parameterized by the rotation matrix ${ }^{C} \mathbf{C}_{C_{\ell}}$ and the translation vector ${ }^{C} \mathbf{t}_{C_{\ell}}$. Thus the projection of ${ }^{C} \mathbf{p}_{f}$ is correlated with the motion of the system (state variables) and therefore imposes constraints on the accumulated INS errors.

By exploiting the fact that the point belongs to the desired plane, ${ }^{C_{\ell}} \mathbf{p}_{f}$ can be expressed in terms of camera pose ${ }^{G} \mathbf{p}_{C_{\ell}}$, $\mathbf{C}\left({ }^{G} \mathbf{q}_{C_{\ell}}\right)$, and observation ${ }^{C_{\ell}} \overline{\mathbf{z}}$. Such a geometric relation is obtained from (12), as

$$
{ }^{C_{\ell}} \mathbf{p}_{f}=\frac{-\mathbf{e}_{3}^{\top} \mathbf{C}\left({ }^{L} \mathbf{q}_{G}\right){ }^{G} \mathbf{p}_{C_{\ell}} C_{\ell}}{\mathbf{e}_{3}^{\top} \mathbf{C}\left({ }^{L} \mathbf{q}_{C_{\ell}}\right)}
$$

Using (24), the general transformation (23) can now be rewritten for ${ }^{C} \mathbf{p}_{f}$ on the defined horizontal plane as

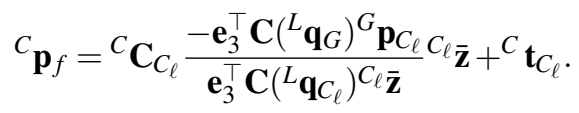

\section{Measurement model}

The observations for the current frame $\{C\}$ are the feature points on the defined plane, matched to those observed in the previous frame $\left\{C_{\ell}\right\}$. Suppose feature point $f_{i}$ has been matched. Then its measured coordinates on the normalized image plane, following (10), is given by

$$
{ }^{C} \mathbf{z}_{i}=\left[\begin{array}{ll}
\mathbf{I}_{2} & \mathbf{0}_{2 \times 1}
\end{array}\right]\left(\mathbf{e}_{3}^{\top C} \mathbf{p}_{f_{i}}\right)^{-1 C} \mathbf{p}_{f_{i}}+\mathbf{n}_{i},
$$

where the pixel noise $\mathbf{n}_{i}$ is assumed to be zero-mean with covariance matrix $\mathbb{C}_{i}=\sigma_{\text {pix }}^{2} \mathbf{I}_{2}$. It is worth mentioning that parametrization of ${ }^{C} \mathbf{p}_{f_{i}}$, in (25), relates the measurement equation to the observation in the previous frame ${ }^{C_{\ell}} \mathbf{z}_{i}$ and camera pose, $\left\{{ }^{G} \mathbf{p}_{C_{\ell}},{ }^{C} \mathbf{q}_{G}\right\}$, both of which are states.

The nonlinear measurement equation constructed from $M$ feature point matches on the same plane is described as

$$
\begin{aligned}
{ }^{C} \mathbf{y}_{M} & =\left[{ }^{C} \mathbf{z}_{1}{ }^{\top} \ldots{ }^{C} \mathbf{z}_{M}{ }^{\top}\right]^{\top} \\
& =\mathbf{h}\left(\delta \mathbf{x}^{\text {ins }}, \delta \mathbf{x}^{\mathrm{cam}},{ }^{C_{\ell}} \overline{\mathbf{y}}_{M}\right)+\mathbf{n}_{M},
\end{aligned}
$$

where

$$
\delta \mathbf{x}^{\mathrm{cam}}=\left[\begin{array}{ll}
\boldsymbol{\delta}^{G} \mathbf{p}_{C_{\ell}} & \delta^{C_{\ell}} \boldsymbol{\theta}_{G}
\end{array}\right]
$$

and ${ }^{C_{\ell}} \overline{\mathbf{y}}_{M}$ are the normalized pixel coordinate of the matched feature in $C_{\ell}$, and the corresponding measurement covariance matrix is $\mathbb{C}_{M}=\sigma_{\mathrm{pix}}^{2} \mathbf{I}_{2} M$.

\section{Motion Estimation}

In this section, we specifically look at the estimation algorithm for the proposed state space formulations in Sections II and III. Equation (6) together with nonlinear equation (27) construct the state space model of the system. To deal with the nonlinearity of the measurement equation with respect to the state variables, we formulate it in the UKF framework as follows.

For the linear process model the covariance matrix propagated as

$$
\mathbf{P}_{k+1}^{-}=\mathbf{F}_{k} \mathbf{P}_{k}^{-} \mathbf{F}_{k}^{\top}+\mathbf{G}_{k} \mathbf{Q} \mathbf{G}_{k}^{\top},
$$

where

$$
\mathbf{F}_{k} \triangleq\left[\begin{array}{cc}
\mathbf{F}_{k}^{\mathrm{ins}} & \mathbf{0} \\
\mathbf{0} & \mathbf{I}_{2 M}
\end{array}\right] \text { and } \mathbf{G}_{k} \triangleq\left[\begin{array}{c}
\mathbf{G}_{k}^{\mathrm{ins}} \\
\mathbf{0}_{2 M \times 12}
\end{array}\right]
$$

When a new image is recorded an error state $\delta^{G} \mathbf{p}_{C_{\ell}}$ and $\delta^{C_{\ell}} \boldsymbol{\theta}_{G}$ are updated into $\delta \mathbf{x}^{\mathrm{cam}}$, and the error covariance matrix is updated as

$$
\mathbf{P}^{\prime}=\left[\begin{array}{cc}
\mathbf{P} & \mathbf{P} \overline{\mathbf{T}}^{\top} \\
\mathbf{\mathbf { T }} \mathbf{P} & \overline{\mathbf{T}} \mathbf{P} \overline{\mathbf{T}}^{\top}
\end{array}\right]
$$

where

$$
\overline{\mathbf{T}}=\left[\begin{array}{cccc}
\mathbf{I}_{3} & \mathbf{0}_{3 \times 3} & \mathbf{0}_{3 \times 3} & \mathbf{0}_{3 \times(6+2 M)} \\
\mathbf{0}_{3 \times 3} & \mathbf{0}_{3 \times 3} & \mathbf{I}_{3} & \mathbf{0}_{3 \times(6+2 M)}
\end{array}\right] .
$$

The UKF is set to perform a measurement update when features on the defined plane have been detected and matched. Based on the state-space model in (27), the UKF estimates the errors linearly as

$$
\delta \hat{\mathbf{x}}_{k}=\mathbf{K}_{k}\left({ }^{C} \mathbf{y}_{k}-{ }^{C} \hat{\mathbf{y}}_{k}\right),
$$

where ${ }^{C} \hat{\mathbf{y}}_{k}$ is the measurement prediction and $\mathbf{K}_{k}$ is the Kalman gain matrix. An overview of the algorithm is given in Algorithm 1.

The joint statistics of $\delta \mathbf{x}$ and $\overline{\mathbf{y}}_{M}$ are propagated as $2 N+$ 1 sigma points, where $N$ is the total number of states $N=$ $15+6+2 M$. Let the joint state vector be denoted by $\mathbf{x}=$ $\left.\left[\begin{array}{ll}\delta \mathbf{x}^{\top} & \overline{\mathbf{y}}_{M}\end{array}\right)^{\top}\right]^{\top}$ and $\hat{\mathbf{x}}^{-}=\left[\begin{array}{ll}\mathbf{0}^{\top} & \widehat{\mathbf{m}}^{\top}\end{array}\right]^{\top}$ be the prediction with error covariance matrix $\mathbf{P}^{-} \oplus \mathbf{C}_{M}$, where $\oplus$ denotes the direct sum of matrices. The sigma points $\mathscr{X}_{j}=\left[\begin{array}{ll}\boldsymbol{\delta} \mathscr{X}_{j}^{\top} & \left.\overline{\mathbf{y}}_{M}\right)_{j}^{\top}\end{array}\right]^{\top}$ are then generated using the matrix square-root of the joint error covariance matrix. By propagating the sigma points through the measurement equation (27), the correlations between the error states $\delta \mathbf{x}$ and observations ${ }^{C} \mathbf{y}$ can be approximated [13]. These correlations form the basis of the Kalman gain.

The weights in the UKF are set as $w_{l}^{c}=w_{l}^{m}=\frac{1}{2(N+\lambda)}$ for $l=1, \ldots, 2 N$. For $l=0, w_{0}^{c}=\frac{\lambda}{N+\lambda}+\left(1-\alpha^{2}+\beta\right)$ and $w_{0}^{m}=$ $\frac{\lambda}{N+\lambda}$. Here $\lambda=\alpha^{2}(N+\kappa)-N$, with parameters set to $\alpha=0.1$, $\beta=2$ and $\kappa=0$ that also determine the spread of the sigma points through the weight $\eta \triangleq \sqrt{N+\lambda}$, [13].

\section{RESUlT}

The performance of the proposed algorithm in the preceding sections was evaluated both in simulations and experiments. Both the simulation and experiments have verified the validity of the 6-DoF motion estimations with high accuracy. Some of the results are given in the following.

\section{A. Simulation results}

For this simulation study, the following settings were considered. A monochrome camera, sampling rate $10 \mathrm{HZ}$, was considered rigidly mounted on top of an IMU, with sampling rate $100 \mathrm{HZ}$. The IMU accelerometers and gyros biases were considered to be $2 \cdot 10^{-3} \mathrm{~m} / \mathrm{s}^{2}$ and $5 \cdot 10^{-4} \mathrm{rad} / \mathrm{s}$ and the standard deviations of the corresponding incremental noise processes, $\mathbf{n}_{a} \mathrm{rad} / \mathrm{s}$ and $\mathbf{n}_{g}$, are $6 \cdot 10^{3} \mathrm{~m} / \mathrm{s}^{2}$ and $3 \cdot 10^{3}$, respectively. Zero-mean white Gaussian noise with standard deviation of 2 pixel was added to the projected image. To evaluate the performance of our planar motion estimation, different planar surfaces were considered for the simulation study. The camera trajectory is the same in all the experiments as is plotted in Fig. 3. We performed four separate experiments 

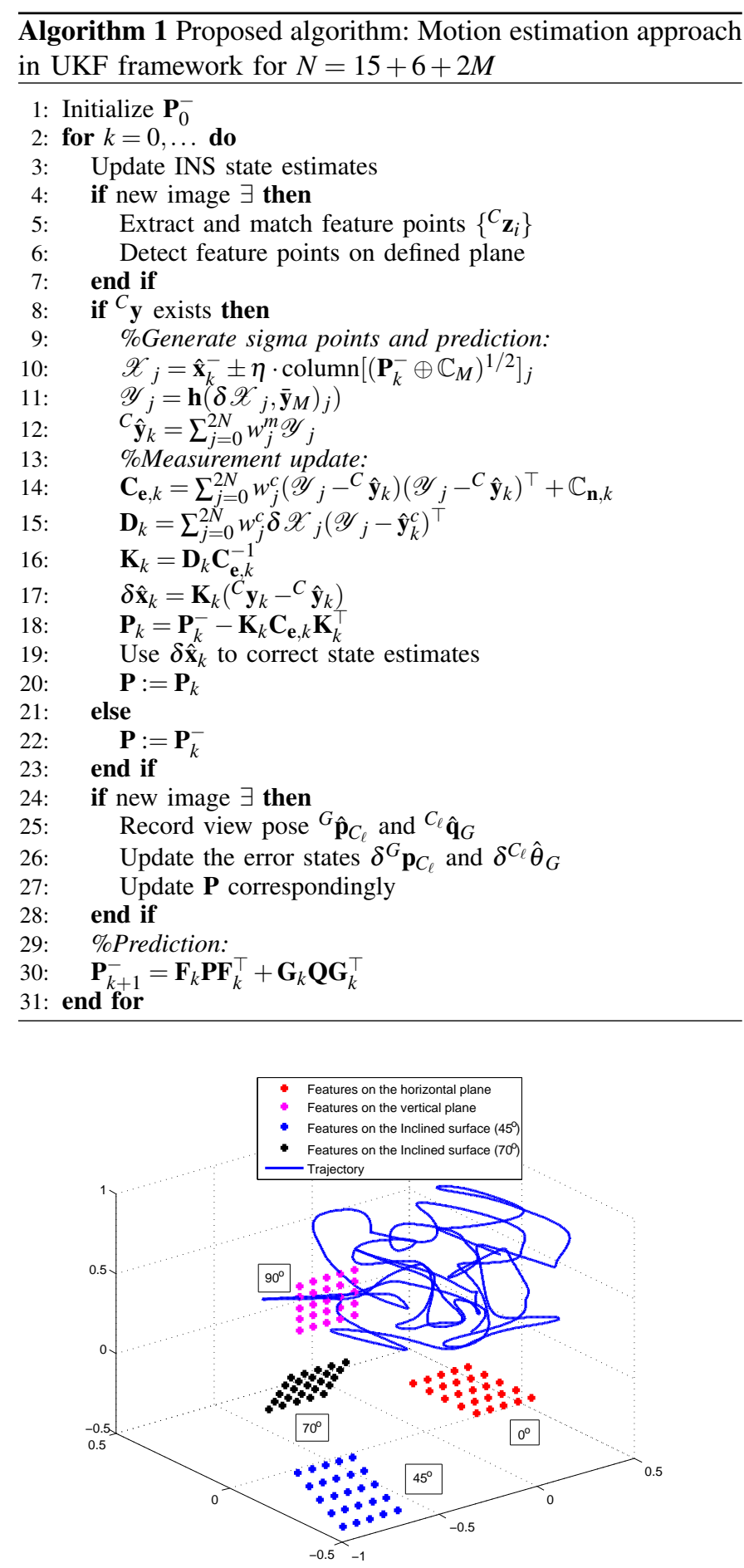

Fig. 3: The 3D trajectory of IMU-camera, and structure of the features on the planes with different slops.

where in each case visual features were assumed to be only in one of the planar surface. We evaluated the estimation accuracy for the planes with slops of $\left\{0^{\circ}, 90^{\circ}, 45^{\circ}, 70^{\circ}\right\}$, see Fig. 3 . For the sake of the visibility, compared to the experiments, planar features are shifted along the axes while keeping the slops.

The estimated positions and velocities for all the four cases along with the ground truth are plotted in Fig. 4 and Fig. 5. As it can be noticed the estimated values are close to the ground truths with the maximum error of about $30 \mathrm{~cm}$ along each axis. To have a better representation of the estimator accuracy, the $\sigma$ bound of the error for the position and attitude are plotted in Fig. 6. The $\sigma$ values are the square root of the corresponding diagonal elements of the states error covariance matrix that provides a representation of the estimation uncertainty. The behaviour of the estimated errors is depicted with different colors for each plane, where the observable states can be distinguished by the bounded errors. As can be seen, considering the position, errors are bounded in the following cases: error along the $y$ axis when navigating in front of the vertical plane $\left(90^{\circ}\right)$, and error along the $z$ axis when navigating in front of the horizontal plane $\left(0^{\circ}\right)$. Considering the rotation, bounded errors are obtained for the roll and pitch for all the planes. Finally, considering the yaw, the error is bounded only when navigating in front of the vertical plane.

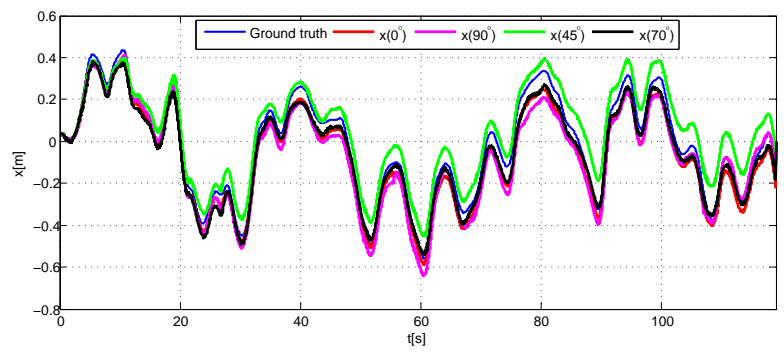

(a) Estimated position along the $x$ axis

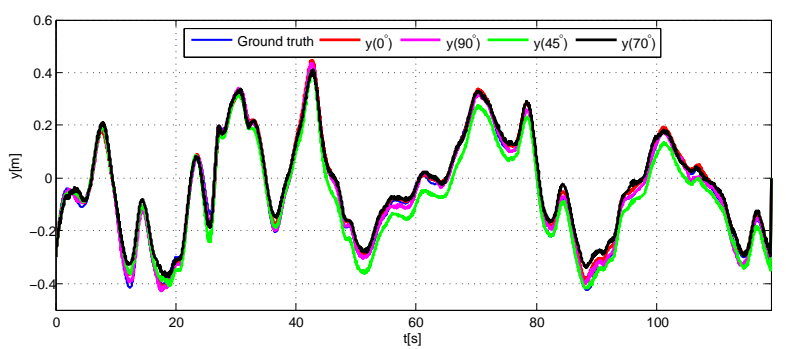

(b) Estimated position along the $y$ axis

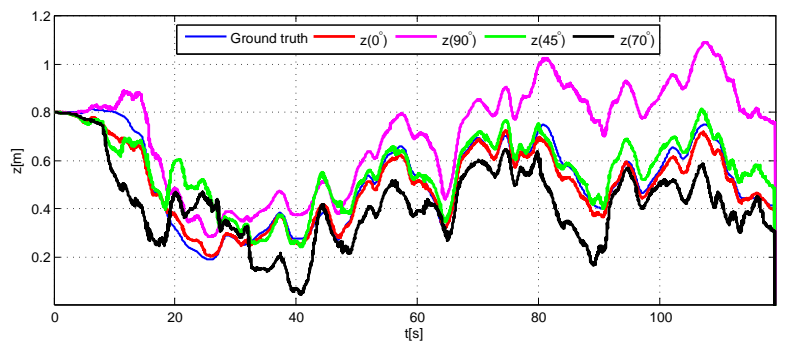

(c) Estimated position along the $z$ axis

Fig. 4: Estimated values of positions for the trajectory of Fig. 3. The IMUcamera is navigating each time in front of a single plane with different alignment. 


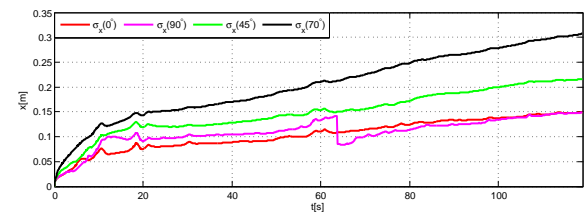

(a)

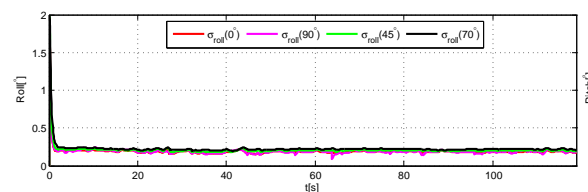

(d)

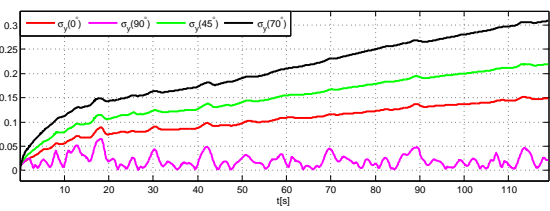

(b)

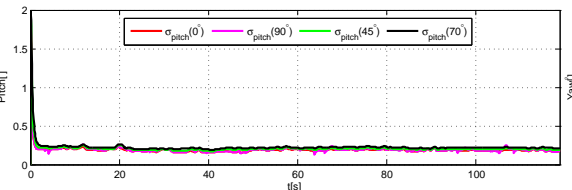

(e)

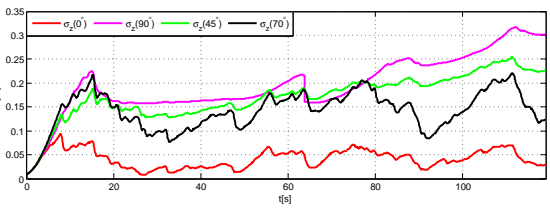

(c)

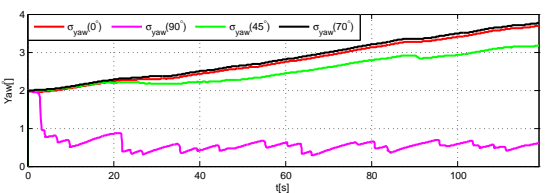

(f)

Fig. 6: The $\sigma$ bounds of the error for (a)-(c) position and (d)-(e) attitude.

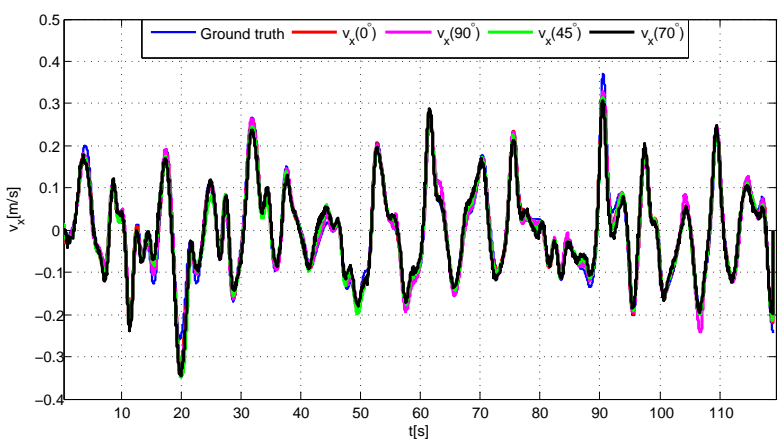

(a) Estimated velocity along the $x$ axis

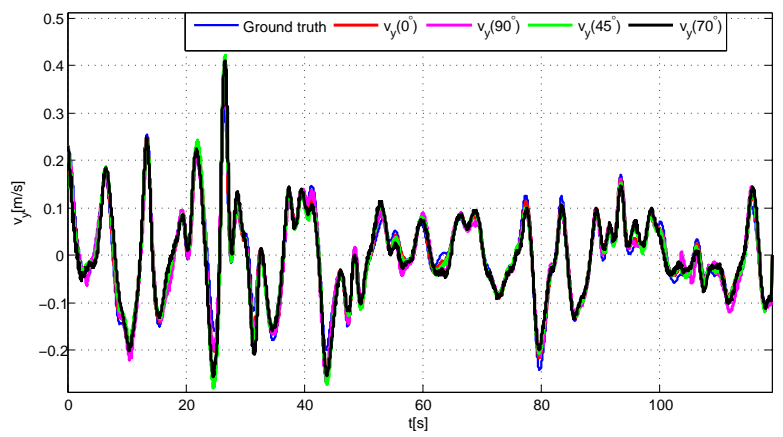

(b) Estimated velocity along the $y$ axis

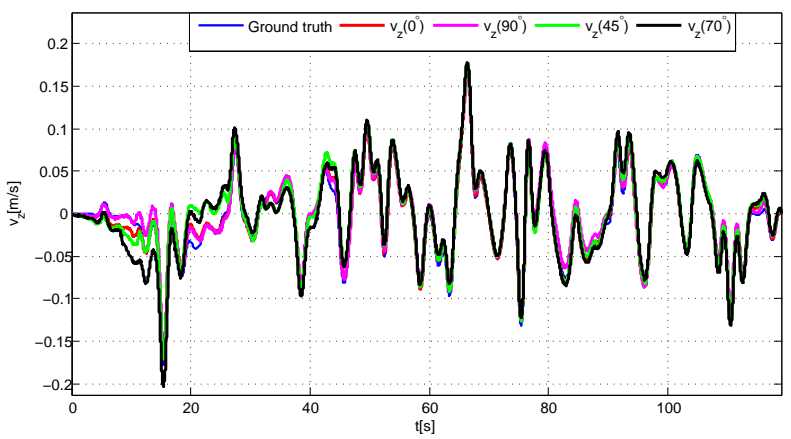

(c) Estimated velocity along the $z$ axis

Fig. 5: Estimated values of velocities for the trajectory of Fig. 3. The IMUcamera is navigating each time in front of a single plane with different alignment.

\section{B. Experimental validation}

In the experiments, an AVT Guppy monochrome camera, with sampling rate of $10 \mathrm{HZ}$, was rigidly mounted on top of a MicroStrain 3DMGX2 IMU, with sampling rate $250 \mathrm{HZ}$. The MATLAB Computer Vision Toolbox implementation of SURF was used for the feature extraction and matching.

An example of the estimated trajectory is plotted in Fig. 7. The traveled path was straight along the $x$ direction for about 15 meters and it was travelled within 90 seconds. For the experiment, the IMU-camera sensor unit was mounted on a cart, measured $100 \mathrm{~cm}$ off the ground, and the camera was observing only features on the vertical plane of a wall with the measured distance of $100 \mathrm{~cm}$ from the camera. For this estimation, maximum 12 features were tracked between two consecutive images. As it can be seen, the final error in the estimated pose along the $x-y$ plane is in the order of centimeter. For the height along the $z$ axis, most of the time the system is capable of error correction that drastically reduces the estimated height error. Moreover, the estimated value of the accelerometer and gyro biases are plotted in Fig. 8a and Fig. 8b. The results show that the estimated value of the biases both for the accelerometers and gyroscopes are approximately stable over time. Additionally, the $\sigma$ bound of the error for the position, velocity, and the attitude for this experiment is plotted in Fig. 9. Expectedly, the positional error along the $y$ and $z$ axes grows slowly as the system has no access to the absolute position update. However, the error along the $x$ axis is bounded (the effect can be more precisely studied through the observability analysis). The $\sigma$ bound error for the velocities, roll, and pitch are bounded since they are observable. And as it is expected the error bound is growing for the yaw, which is rotation around the gravity.

In another experiment the reliability of the estimated height was tested by placing the IMU-camera on a test table where the system was only moving up and down parallel to the gravity and the camera was observing features on the horizontal plane. The maximum number of tracked features between two consecutive image was set to 5 . The $3 \mathrm{D}$ estimated position is shown in Fig. 10. The system was lifted up and down, 

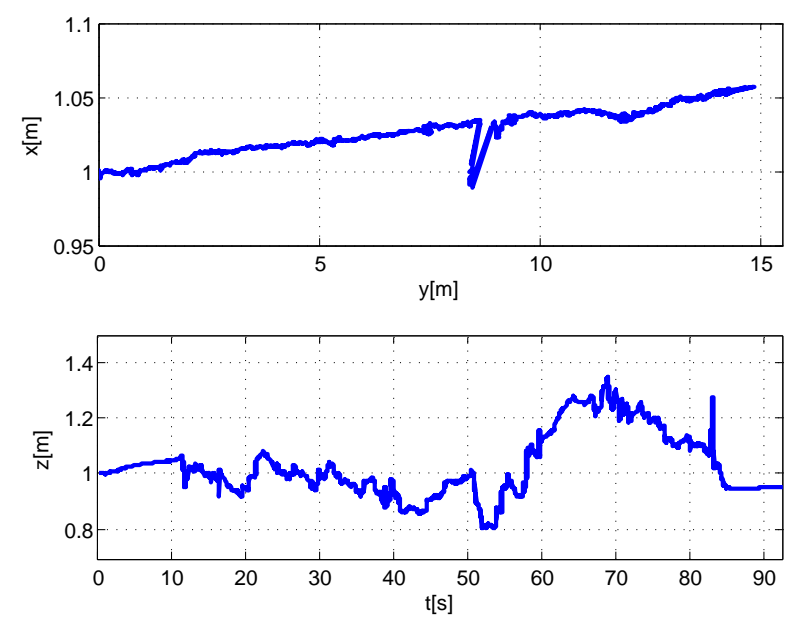

Fig. 7: Estimated position along the $x-y$ plane and $z$ axis.

to approximately same height, by a step motor for about 110 seconds. The initial and the final measured height was approximately $100 \mathrm{~cm}$ and $95 \mathrm{~cm}$, respectively, and height of the measured peak was about $155 \mathrm{~cm}$. As it is shown, the estimated error along the $x$ and $y$ directions was in the order of centimeter. For this estimate, the maximum number of features, used for estimation, was set to 7 between two successive images.

\section{DISCUSSION}

A vision-aided inertial navigation system is proposed in which the camera measurements are projections of planar features in 2D image planes. The main idea of this study was to devise a simple 6-DoF motion estimation with low computational complexity, in which only planar features are used for motion estimation. From the filter perspective, the length of the state vector is only $15+6$ (ignoring the future point measurements which are only nuisance parameters), that avoids the complexity of calculating the inverse of the covariance matrices.

The performance of the proposed method was evaluated using both computer simulations and experiments. For the simulation, multiple planes with different slopes $\left\{0^{\circ}, 90^{\circ}, 45^{\circ}, 70^{\circ}\right\}$ were tested and the estimated errors were in the order of centimetres, which indicates the reliability of the proposed method. In the experiments, we only considered horizontal and vertical planes with known normals. The assumption of knowing the plane normal is a practical assumption in some applications, e.g., in robot manipulators where the navigation is over a tilted platform and the alignment of the navigation platform is known in advance. As a feature work, we are also considering to generalize the system where the normal of the plane can be simultaneously estimated with the rest of the state variables.

Finally, the observability analysis for the proposed model is suggested as a future work. Such analysis can reveal interesting results regarding the observable directions along the normal of the plane [9].
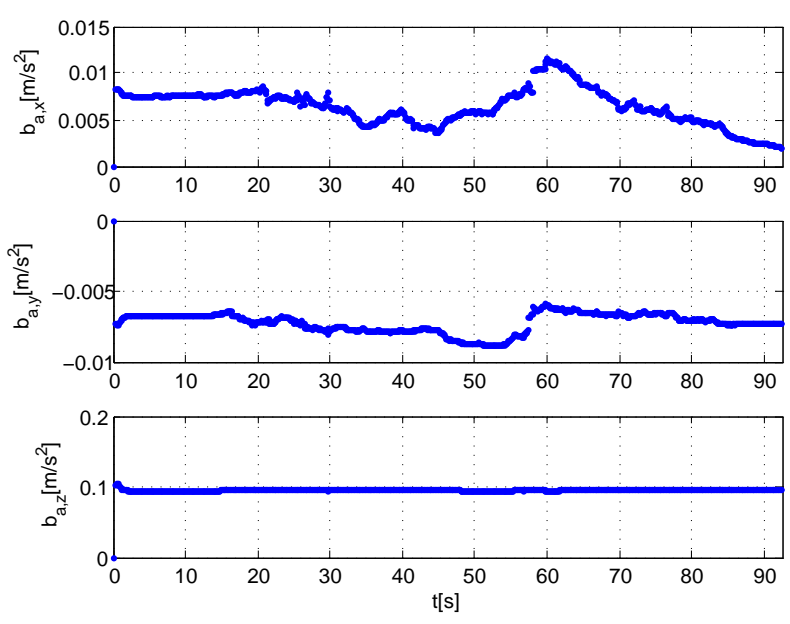

(a)
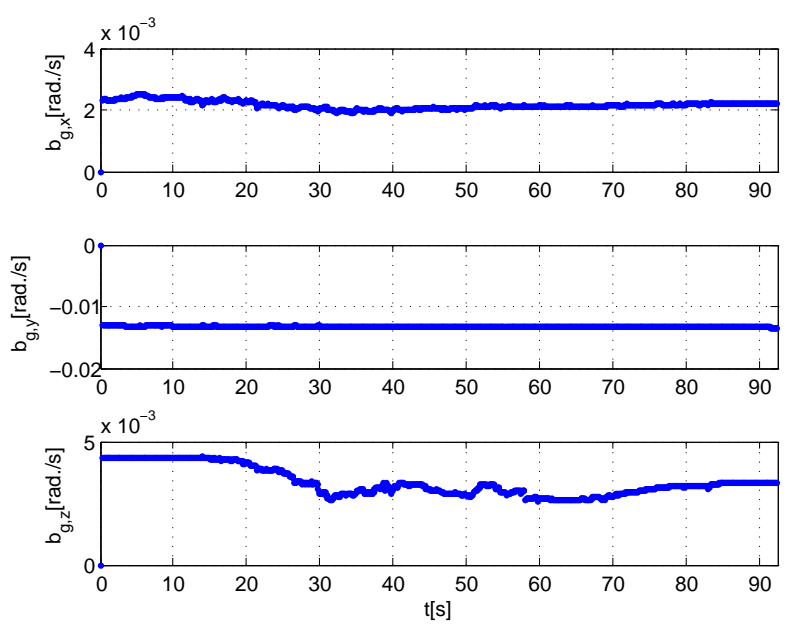

(b)

Fig. 8: The estimated (a) accelerometer biases and (c) gyro biases.

\section{CONCLUSION}

In this paper, a visually aided inertial navigation system is introduced for 3D motion estimation. The estimation is formulated in the UKF framework in which the propagations of the states are calculated from an IMU inertial data and the estimation error is corrected periodically using the planar visual features observed from a camera. The proposed new measurement model is not restricted for a horizontal plane, as the existing models are, but it is generalized for arbitrary planar surfaces. The mathematical derivations in this paper are extensively evaluated where the reported results, both from the simulations and experiments, verify the validity of the 6-DoF motion estimation with high accuracy.

\section{REFERENCES}

[1] A. I. Mourikis, N. Trawny, S. I. Roumeliotis, A. E. Johnson, A. Ansar, and L. Matthies, "Vision-aided inertial navigation for spacecraft entry, descent, and landing," IEEE Trans. on Robotics, vol. 25, no. 2, pp. 264280, 2009.

[2] C. Morse, A. Ziegler, D. Gilbert, and A. Jones, "Autonomous surface cleaning robot for dry cleaning," Jun. 2014. US Patent 8,739,355. 

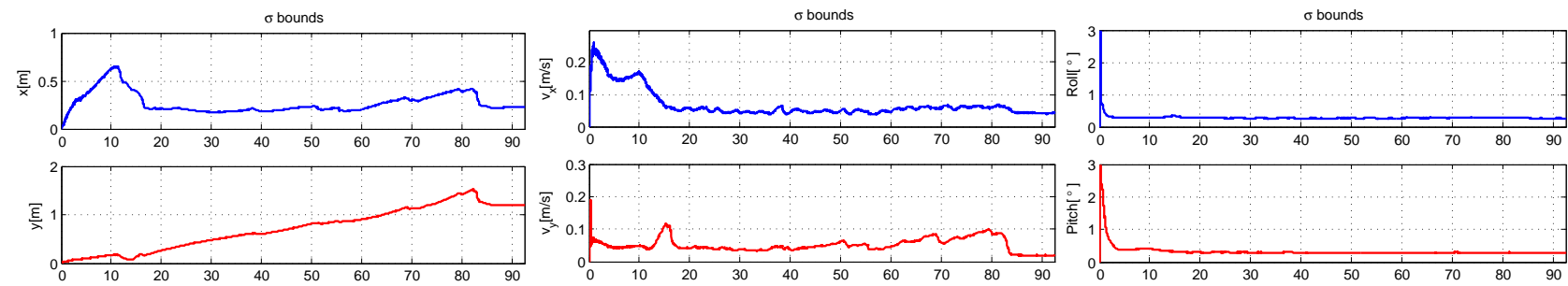

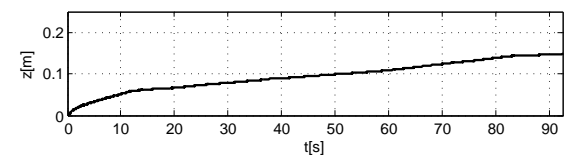

(a) The $\sigma$ bounds error for position

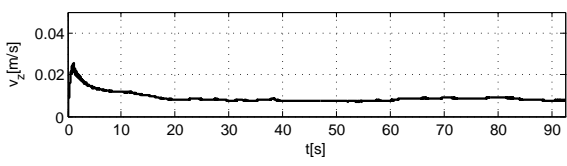

(b) The $\sigma$ bounds error for velocity

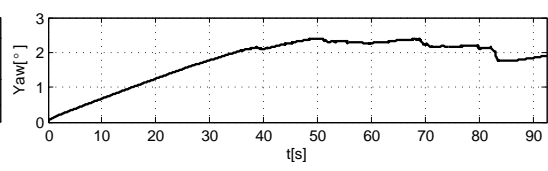

(c) The $\sigma$ bounds error for attitude

Fig. 9: The $\sigma$ bounds of the error for the (a) position, (b) velocity, and (c) attitude.
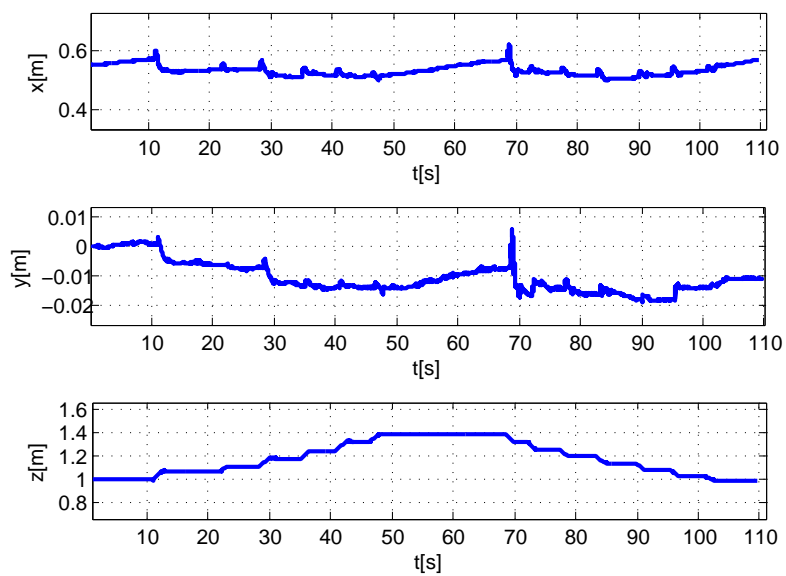

Fig. 10: Estimated position along the $x, y$, and $z$ axes for the experiment in which the IMU-camera sensor was lifted up and down vertically.

[3] N. Kaempchen, U. Franke, and R. Ott, "Stereo vision based pose estimation of parking lots using $3 \mathrm{~d}$ vehicle models," in IEEE Intelligent Vehicle Symposium, vol. 2, pp. 459-464, 2002.

[4] T. Kubota, I. Nakatani, K. Watanabe, and S. Shimoda, "Study on moletyped deep driller robot for subsurface exploration," in IEEE International Conference on Robotics and Automation (ICRA), pp. 1297-1302, IEEE, 2005.

[5] X. Song, L. D. Seneviratne, and K. Althoefer, "A Kalman filterintegrated optical flow method for velocity sensing of mobile robots," IEEE/ASME Trans. on Mechatronics, vol. 16, no. 3, pp. 551-563, 2011.

[6] S. I. Roumeliotis, A. E. Johnson, and J. F. Montgomery, "Augmenting inertial navigation with image-based motion estimation," in Proc. IEEE Int. Conf. on Robotics and Automation (ICRA), Washington D.C., pp. 4326-4333, May 11-15, 2002.

[7] D. Conrad and G. DeSouza, "Homography-based ground plane detection for mobile robot navigation using a modified em algorithm," in Proc. IEEE Int. Conf. on Robotics and Automation (ICRA), Anchorage, Alaska, pp. 910-915, May 3-8, 2010.

[8] N. Pears and B. Liang, "Ground plane segmentation for mobile robot visual navigation," in Proc. IEEE/RSJ Int. Conf. on Intelligent Robots and Systems (IROS), Outrigger Wailea Resort, Maui, Hawaii, pp. 15131518,, Oct.29-Nov.3, 2001.

[9] G. Panahandeh, Selected Topics in Inertial and Visual Sensor Fusion : Calibration, Observability Analysis and Applications. $\mathrm{PhD}$ thesis, KTH, Signal Processing, 2014. QC 20140312.

[10] V. Pradeep, K. Konolige, and E. Berger, "Calibrating a multi-arm multisensor robot: A bundle adjustment approach," in Experimental Robotics, pp. 211-225, Springer, 2014.

[11] J. A. Farrell and M. Barth, Global Positioning System, Inertial Naviga- tion and Integration. McGraw-Hill Companies, 1999.

[12] R. I. Hartley and A. Zisserman, Multiple View Geometry in Computer Vision. Cambridge University Press, ISBN: 0521623049, 2000.

[13] S. J. Julier and J. K. Uhlmann, "A new extension of the Kalman filter to nonlinear systems," in Proc. of Signal Processing, Sensor fusion, and Target Recognition, vol. 4, pp. 182-193, Apr. 1997. 